\title{
Magnetoacoustic Waves in Relaxation Model of Magnetizable Liquids
}

\author{
V A Zhelnorovich \\ Research Institute of Mechanics, Moscow State University, Moscow, 119192 \\ *Corresponding Author: v.zhelnor@imec.msu.ru
}

Copyright (C2014 Horizon Research Publishing All rights reserved.

\begin{abstract}
Within the framework of the relaxation model, the solutions describing transverse and longitudinal magnetoacoustic waves are obtained for magnetizable liquids with an arbitrary orientation of the wave vector and the magnetization vector. It is shown that two different transverse waves exist in magnetizable liquids according to the relaxation model. Limitations are established on the internal liquid energy at which the velocity of sound is decreased from the maximum value when the wave is propagated along the magnetization vector to the minimum value when the wave is propagated perpendicular to the magnetization vector. Conditions are also established at which the velocity of sound, according to the experimental data, increases as the liquid magnetization and wave frequency increase.
\end{abstract}

Keywords Magnetizable Liquids, Magnetoacoustic Waves

PACS number 47.65.- $\mathrm{Cb}, 75.50 .-\mathrm{Mm}$

\section{Introduction}

The simplest model of the isotropic continuum taking into consideration its magnetization, is based on the equation $\boldsymbol{M}=\chi \boldsymbol{H}$ describing the relationship between the magnetization volume density vector $\boldsymbol{M}$ and the magnetic field strength vector $\boldsymbol{H}, \chi$ is magnetic susceptibility. The magnetizable liquid (ML) model based on such an equation was introduced by Neuringer and Rosensweig [1]. Such a model is now used and satisfactorily describes different simple motions of ML which is a finely dispersed system of single-domain ferromagnetic particles in liquid. However, there are real effects and phenomena (for example, increase of viscosity in the magnetic field, entrainment of ML by a rotating magnetic field, propagation of high-frequency waves in ML, etc.) which are not described at all, or are described incorrectly by the Neuringer-Rosensweig model. Some such effects were described within the framework of the model with internal rotation [2] which gained currency in $70 \mathrm{~s}$ and $80 \mathrm{~s}$ of the last century. However, in $[3,4]$ it was shown that the description of such phenomena is not associated in fact with the concept of internal rotation. Besides, the theory related to internal rotation accounted for the internal angular momentum of the ML caused by the internal rotation, while the significantly greater (by many orders more) internal spin momentum existing in real ML was not taken into account. Hence, the theory of ML with internal rotation is no longer used and is interesting only historically.

The modern models of ML adjusting the NeuringerRosensweig model are mainly related to consideration of the processes of relaxation in the equation for the magnetization vector. For ML at rest the equation for magnetization taking into account relaxation processes can be represented as (the similar equations were considered in [5])

$$
H^{\alpha}-\frac{\partial U_{m}}{\partial m_{\alpha}}=\rho \theta^{\alpha \beta} \frac{\partial m_{\beta}}{\partial t} .
$$

Here $U_{m}$ is the specific density of the internal energy of the liquid, $\rho$ is the mass density of the liquid, $m_{\alpha}=$ $M_{\alpha} / \rho$ are the components of the magnetization specific density vector of the liquid; $H^{\alpha}$ are the components of the magnetic field strength vector; $t$ is the time; $\theta^{\alpha \beta}$ are given coefficients.

The question now arises of as to what is equation (1) for moving ML. Different variants of such notation are possible. One of the natural variants of the equations for magnetization for arbitrary moving ML can be equations set down in comoving system of coordinates as

$\widehat{B}_{\alpha}-\frac{\partial F}{\partial \widehat{m}^{\alpha}}=\rho \widehat{\theta}_{\alpha \beta} \frac{d \widehat{m}^{\beta}}{d t} \quad$ or $\quad \widehat{B}^{\alpha}-\frac{\partial F}{\partial \widehat{m}_{\alpha}}=\rho \widehat{\theta}^{\alpha \beta} \frac{d \widehat{m}_{\beta}}{d t}$,

where $F$ is the specific density of the free energy of the liquid; symbol ${ }^{\wedge}$ means that the components marked by it are calculated in comoving system of coordinates. The total time derivative $d / d t$ is calculated with the constant variables of the comoving system of coordinates. This equation is a special case of more general equations taking into account both magnetization and polarization of the continuous medium considered in [6].

In the inertial coordinate system equations (2) are rep- 
resented, respectively, as follows

$$
\begin{aligned}
& B_{\alpha}-\frac{\partial F}{\partial m^{\alpha}}=\rho \theta_{\alpha \beta}\left(\frac{d m^{\beta}}{d t}-m^{\lambda} \nabla_{\lambda} v^{\beta}\right), \\
& B^{\alpha}-\frac{\partial F}{\partial m_{\alpha}}=\rho \theta^{\alpha \beta}\left(\frac{d m_{\beta}}{d t}+m_{\lambda} \nabla_{\beta} v^{\lambda}\right) .
\end{aligned}
$$

Here $\nabla_{\alpha}$ is the symbol of the covariant derivative relative to the inertial system of coordinates. $v^{\alpha}$ are the components of the velocity vector of points in the medium.

Instead of $(3)$ in $[3,4]$ the following equations were used for description of magnetization:

$$
\begin{gathered}
H^{\alpha}-\frac{\partial U_{m}}{\partial m_{\alpha}}-\gamma \omega^{\alpha}=\rho \theta^{\alpha \beta}\left(\frac{d m_{\beta}}{d t}-[\boldsymbol{\omega}, \boldsymbol{m}]_{\beta}\right) \\
\equiv \rho \theta^{\alpha \beta}\left[\frac{d}{d t} m_{\beta}+\frac{1}{2} m^{\mu}\left(\nabla_{\beta} v_{\mu}-\nabla_{\mu} v_{\beta}\right)\right],
\end{gathered}
$$

where $\gamma$ is a constant; $\boldsymbol{\omega}=\left\{\omega^{\alpha}\right\}=\frac{1}{2} \operatorname{curl} \boldsymbol{v}$ is the vorticity vector; the gyromagnetic term $-\gamma \omega^{\alpha}$ in this equation corresponds to the Lagrangian of matter $\Lambda=$ $\rho U_{m}+\gamma \rho m_{\alpha} \omega^{\alpha}$. Equations (1) when $\gamma=0$ thereafter were considered, for instance, in [7].

One can consider also the alternative equation for the magnetization volume density

$$
\begin{gathered}
H^{\alpha}-\frac{\partial U_{m}}{\partial m_{\alpha}}-\gamma \omega^{\alpha}=\theta^{\alpha \beta}\left(\frac{d M_{\beta}}{d t}-[\boldsymbol{\omega}, \boldsymbol{M}]_{\beta}\right) \\
\equiv \theta^{\alpha \beta}\left[\frac{d}{d t} M_{\beta}+\frac{1}{2} M^{\mu}\left(\nabla_{\beta} v_{\mu}-\nabla_{\mu} v_{\beta}\right)\right] .
\end{gathered}
$$

Let us write out also a relaxation equation [2] obtained as an approximate equation corresponding to the equations in ML theory with internal rotation $\left(\tau_{B}, \tau_{s}\right.$ are relaxation time values, $I$ is constant):

$$
\frac{d \boldsymbol{M}}{d t}=[\boldsymbol{\omega}, \boldsymbol{M}]-\frac{1}{\tau_{B}}\left(\boldsymbol{M}-M_{0} \frac{\boldsymbol{H}}{H}\right)-\frac{\tau_{s}}{I}[\boldsymbol{M},[\boldsymbol{M}, \boldsymbol{H}]] .
$$

This equation supposes the existence of the equilibrium value of magnetization $M_{0}$ and thus it may only have limited application.

The choice of which of the equations for magnetization should be used must be based on the solution of particular problems and comparison with the experiment. As is known, the viscosity of an ML in a magnetic field increases. It can be shown (it is beyond the scope of the article) that the change of the ML viscosity in the magnetic field in solving the Poiseuille problem on motion of ML in cylindrical pipes using the first and the second equations in (3) does not correspond with the known $[8,9]$ experimental data. The ML model with relaxation equation (1) or (1) with the respective setting of relaxation times describes the change of viscosity in the magnetic field correctly. Hence, the most acceptable equations are (1) or (1) in which a small gyromagnetic term $-\gamma \omega^{\alpha}$ can be neglected.

\section{Relaxation model of magnetiz- able liquids.}

Further let us consider the following system of equations determining the model of a magnetizable isotropic liquid in a magnetic field in the magnetostatic approximation

$$
\begin{gathered}
\rho \frac{d}{d t} v^{\alpha}=-\nabla^{\alpha} p+\frac{1}{2}\left(M^{\beta} \nabla^{\alpha} H_{\beta}-H_{\beta} \nabla^{\alpha} M^{\beta}\right) \\
+\frac{1}{2} \nabla_{\beta}\left(M^{\alpha} H^{\beta}-M^{\beta} H^{\alpha}\right)+\nabla_{\beta} \tau^{\alpha \beta}+Q^{\alpha}, \\
\frac{d}{d t} \rho+\rho \nabla_{\alpha} v^{\alpha}=0, \\
\nabla_{\alpha}\left(H^{\alpha}+4 \pi M^{\alpha}\right)=0, \quad \nabla_{\alpha} H_{\beta}-\nabla_{\beta} H_{\alpha}=0, \\
H^{\alpha}-\frac{1}{\chi} M^{\alpha}=L^{\alpha}, \quad \frac{1}{\chi}=\frac{\rho}{M} \frac{\partial U_{m}}{\partial M}, \\
T=\frac{\partial U_{m}}{\partial s}, \quad \rho T \frac{d s}{d t}=-\nabla_{\alpha} q^{\alpha}+\tau^{\alpha \beta} \nabla_{\alpha} v_{\beta} \\
+L^{\alpha}\left[\frac{d}{d t} M_{\alpha}+\frac{1}{2} M^{\mu}\left(\nabla_{\alpha} v_{\mu}-\nabla_{\mu} v_{\alpha}\right)\right] .
\end{gathered}
$$

Here $\nabla_{\alpha}$ is the symbol of the covariant derivative relative to the inertial system of coordinates with variable $x^{\alpha}, \alpha=1,2,3$, relative to which all vectors and tensors in equations (7) are calculated; $Q^{\alpha}$ are the components of the vector of the external force acting on the liquid (e.g., the gravity force); $M^{\alpha}=\rho m^{\alpha}$ are the components of the magnetization volume density vector of the liquid; $s$ is the specific entropy density; $T$ is the quantity playing the role of temperature in equilibrium processes; the specific density of the internal energy of the liquid $U_{m}$ is assumed to be a given function of density $\rho$ of the liquid, specific entropy density $s$, and modulus $M$ of the magnetization vector:

$$
U_{m}=U_{m}(\rho, s, M), \quad M=\left(M_{\alpha} M^{\alpha}\right)^{1 / 2} .
$$

Pressure $p$ in the equation of momenta in system (7) is defined as

$$
p=\rho^{2} \frac{\partial U_{m}}{\partial \rho}+\frac{1}{2} M_{\alpha} H^{\alpha}
$$

Symmetric components of the viscous stress tensor $\tau^{\alpha \beta}=\tau^{\beta \alpha}$ and components of the heat flux density vector $q^{\alpha}$ in equations (7) we will define by the relations

$$
\tau^{\alpha \beta}=\eta\left(\nabla^{\alpha} v^{\beta}+\nabla^{\beta} v^{\alpha}\right)+\lambda g^{\alpha \beta} \nabla_{\gamma} v^{\gamma}, \quad q^{\alpha}=-\kappa \nabla^{\alpha} T,
$$

in which $g^{\alpha \beta}$ are the metric tensor components in the system of coordinates with variables $x^{\alpha}, \lambda$ and $\eta$ are the viscosity coefficients, $\kappa$ is the thermal conductivity.

The relaxation term $L^{\alpha}$ in equations (7) are chosen in such a way so that to ensure the positivity of the inner production of entropy. In particular, we can determine $L^{\alpha}$ as

$$
L^{\alpha}=\theta^{\alpha \beta}\left[\frac{d}{d t} M_{\beta}+\frac{1}{2} M^{\mu}\left(\nabla_{\beta} v_{\mu}-\nabla_{\mu} v_{\beta}\right)\right],
$$

where $\theta^{\alpha \beta}$ are fixed positive definite quadratic forms. For isotropic ML it can be assumed that

$$
\theta^{\alpha \beta}=\theta_{\perp}\left(g^{\alpha \beta}-\frac{1}{M^{2}} M^{\alpha} M^{\beta}\right)+\frac{\theta_{\|}}{M^{2}} M^{\alpha} M^{\beta} .
$$

The relaxation times $\theta_{\perp}$ and $\theta_{\|}$may be functions of the determining parameters of liquid and field and should be set. 
Replacing in equations (7) quantities $L^{\alpha}$ via field $H^{\alpha}$ are given by equations (9), the equations for magnetization can be written in the form

$$
H^{\alpha}-\frac{1}{\chi} M^{\alpha}=\theta^{\alpha \beta}\left[\frac{d}{d t} M_{\beta}+\frac{1}{2} M^{\mu}\left(\nabla_{\beta} v_{\mu}-\nabla_{\mu} v_{\beta}\right)\right] \text {. }
$$

In momentum equation (7) terms $\frac{1}{2}\left(M_{\beta} \nabla^{\alpha} H^{\beta}-\right.$ $\left.H^{\beta} \nabla^{\alpha} M_{\beta}\right)$ determine the vector of the ponderomotive force acting on the liquid from the magnetic field and is represented as a divergence of the Maxwell tensor

$$
\begin{aligned}
\frac{1}{2}\left(M_{\beta} \nabla^{\alpha} H^{\beta}-H^{\beta} \nabla^{\alpha} M_{\beta}\right) & \\
& =\frac{1}{4 \pi} \nabla_{\beta}\left(H^{\alpha} B^{\beta}-\frac{1}{2} g^{\alpha \beta} B_{\lambda} H^{\lambda}\right) .
\end{aligned}
$$

The total stress tensor of the liquid and field is determined by symmetric components

$\sigma^{\alpha \beta}=-p g^{\alpha \beta}+\tau^{\alpha \beta}+\frac{1}{8 \pi}\left(B^{\alpha} H^{\beta}+B^{\beta} H^{\alpha}-g^{\alpha \beta} B_{\lambda} H^{\lambda}\right)$

and satisfy the momentum equation

$$
\rho \frac{d}{d t} v^{\alpha}=\nabla_{\beta} \sigma^{\alpha \beta}+Q^{\alpha}
$$

System of equations (7) - (13) defines the relaxation model of a magnetizable liquid. This can be used e.g., for describing ferrofluids. Derivation of equations (7)-(13) and more general equations using the variation principle is provided in [4]. Equations (7) for relaxation term equal to zero $L^{\alpha}=0$ (in this case the divergence term $\frac{1}{2} \nabla_{\beta}\left(M^{\alpha} H^{\beta}-M^{\beta} H^{\alpha}\right)$ in the momentum equation is also equal to zero) were considered in [10].

Further on we will consider adiabatic motions of a magnetizable liquid described by equations (7), (9) without external forces $Q^{\alpha}=0, s=s_{0}=$ const, without thermal conductance $q^{\alpha}=0$ and with constant relaxation times $\theta_{\|}, \theta_{\perp}$. We assume that the system of coordinates with variables $x^{\alpha}$ is inertial and Cartesian, and quantities $\rho, M^{\alpha}, h^{\alpha}$ and $v^{\alpha}$ in equations (7) change insignificantly relative to their constant equilibrium values $\rho_{0}, M_{0}^{\alpha}, h_{0}^{\alpha}$ and $v_{0}^{\alpha}=0$. We put

$$
\rho=\rho_{0}+\rho_{1}, \quad M^{\alpha}=M_{0}^{\alpha}+\mu^{\alpha}, \quad H^{\alpha}=H_{0}^{\alpha}+h^{\alpha},
$$

where $\rho_{1}, \mu^{\alpha}, h^{\alpha}$ and velocity vector components $v^{\alpha}$ are small quantities treated as first-order infinitesimals.

Function $U_{m}(\rho, s, M)$ to within second-order terms can be represented as

$$
\begin{aligned}
\rho U_{m}=\frac{1}{2 \rho_{0}} a_{*}^{2} \rho_{1}^{2}+\frac{1}{2} \beta^{\alpha \lambda} \mu_{\alpha} \mu_{\lambda}+b \nu^{\alpha} \mu_{\alpha} \rho_{1} \\
+D \nu^{\alpha} \mu_{\alpha}+A \rho_{1}+\text { const. }
\end{aligned}
$$

Here $\nu^{\alpha}=M_{0}^{\alpha} / M_{0}$ are the components of the unit vector directed along magnetization vector. The tensor components $\beta^{\alpha \lambda}$ are determined by the relation

$$
\beta^{\alpha \lambda}=\beta_{\perp}\left(g^{\alpha \lambda}-\nu^{\alpha} \nu^{\lambda}\right)+\beta_{\|} \nu^{\alpha} \nu^{\lambda} .
$$

The coefficients $a_{*}, b$ and $\beta$ are expressed in terms of the internal energy $U_{m}$ as:

$$
\begin{gathered}
a_{*}^{2}=\left(\rho \frac{\partial^{2} \rho U_{m}}{\partial \rho^{2}}\right)_{0}, \quad b=\left(\frac{\partial^{2} \rho U_{m}}{\partial \rho \partial M}\right)_{0} \equiv\left(M \frac{\partial}{\partial \rho} \frac{1}{\chi}\right)_{0}, \\
\beta_{\|}=\left(\frac{\partial^{2} \rho U_{m}}{\partial M^{2}}\right)_{0} \equiv\left(\frac{1}{\chi}+M \frac{\partial}{\partial M} \frac{1}{\chi}\right)_{0} \\
\beta_{\perp}=\left(\frac{\rho}{M} \frac{\partial U_{m}}{\partial M}\right)_{0} \equiv\left(\frac{1}{\chi}\right)_{0} .
\end{gathered}
$$

Here the magnetic susceptibility $\chi$ is defined by equations (7); quantities in parentheses $(\ldots)_{0}$ are evaluated for constant equilibrium values of parameters $\rho=\rho_{0}$, $s=s_{0}$ and $M=M_{0}$.

Taking into account equations (9) and (10) we linearize equations (7). This yields

$$
\begin{gathered}
\rho_{0} \frac{\partial}{\partial t} v_{\alpha}=-a_{*}^{2} \partial_{\alpha} \rho_{1}-\rho_{0} b \nu_{\beta} \partial_{\alpha} \mu^{\beta} \\
+\frac{1}{2} M_{0} \partial_{\beta}\left[\nu_{\alpha}\left(h^{\beta}-\beta_{\perp} \mu^{\beta}\right)-\nu^{\beta}\left(h_{\alpha}-\beta_{\perp} \mu_{\alpha}\right)\right] \\
+\eta \Delta v_{\alpha}+(\lambda+\eta) \partial_{\alpha} \partial_{\beta} v^{\beta} \\
\partial_{\alpha}\left(h^{\alpha}+4 \pi \mu^{\alpha}\right)=0, \quad \partial_{\alpha} h_{\beta}-\partial_{\beta} h_{\alpha}=0 \\
h^{\alpha}=\beta^{\alpha \lambda} \mu_{\lambda}+\nu^{\alpha} b \rho_{1}+\theta^{\alpha \beta} \frac{\partial}{\partial t} \mu_{\beta} \\
+\frac{1}{2} M_{0} \theta_{\perp} \nu_{\beta}\left(\partial^{\alpha} v^{\beta}-\partial^{\beta} v^{\alpha}\right) \\
\partial \rho_{1} / \partial t+\rho_{0} \partial_{\alpha} v^{\alpha}=0
\end{gathered}
$$

Here $\partial_{\alpha}=\partial / \partial x^{\alpha} ; \Delta=g^{\alpha \beta} \partial_{\alpha} \partial_{\beta}$ is the Laplace operator.

\section{Plane harmonic waves in mag- netizable liquids.}

We will seek a solution of equations (15) in the form of plane harmonic waves, for which all unknown functions vary in accordance with the law $\exp i\left(k_{\alpha} x^{\alpha}-\omega t\right)$, where $\mathrm{i}=\sqrt{-1}, \omega$ and $k_{\alpha}$ are constants. Excluding quantities $\rho_{1}$ and $h^{\alpha}$ from equations (15) using the Maxwell equations and equation of continuity, we obtain the system of equations

$$
\begin{gathered}
\left\{\left(\omega \rho_{0}+i \eta k^{2}\right) \delta_{\beta}^{\alpha}+\left[-\frac{\rho_{0}}{\omega} a_{*}^{2}+i(\lambda+\eta)\right] k^{\alpha} k_{\beta}\right\} v^{\beta} \\
+\frac{1}{2} M_{0}\left[-\left(4 \pi+\beta_{\perp}\right) \nu^{\alpha}+\frac{4 \pi}{k} \cos \psi_{0} k^{\alpha}\right] k_{\beta} \mu^{\beta} \\
-\rho_{0} b k^{\alpha} \nu_{\beta} \mu^{\beta}+\frac{1}{2} \beta_{\perp} M_{0} k \cos \psi_{0} \mu^{\alpha}=0 \\
{\left[\left(\beta_{\perp}-i \omega \theta_{\perp}\right)\left(\delta_{\beta}^{\alpha}-\nu^{\alpha} \nu_{\beta}\right)+\left(\beta_{\|}-i \omega \theta_{\|}\right) \nu^{\alpha} \nu_{\beta}\right.} \\
\left.+\frac{4 \pi}{k^{2}} k^{\alpha} k_{\beta}\right] \mu^{\beta}+\left[\rho_{0} \frac{b}{\omega} \nu^{\alpha} k_{\beta}\right. \\
\left.+\frac{i}{2} M_{0} \theta_{\perp}\left(k^{\alpha} \nu_{\beta}-k \cos \psi_{0} \delta_{\beta}^{\alpha}\right)\right] v^{\beta}=0
\end{gathered}
$$

where $k=\left(k_{\alpha} k^{\alpha}\right)^{1 / 2}$ is the wave number; $\delta_{\beta}^{\alpha}$ are the Kronecker deltas; $\psi_{0}$ is the angle between the wave vector and the magnetization vector, $\cos \psi_{0}=k_{\alpha} M_{0}^{\alpha} /\left(k M_{0}\right)=$ $k_{\alpha} \nu^{\alpha} / k$.

First let us consider the case where $\sin \psi_{0}=0$, i. e. the wave vector is collinear with the magnetization vector $k^{\alpha}= \pm k \nu^{\alpha}$. In this case the system of equations (16) 
splits into a system of equations for quantities $\nu_{\alpha} \mu^{\alpha}$ and $k_{\alpha} v^{\alpha}$ :

$$
\begin{gathered}
\left(4 \pi+\beta_{\|}-i \omega \theta_{\|}\right) \nu_{\alpha} \mu^{\alpha}+\rho_{0} \frac{b}{\omega} k_{\alpha} v^{\alpha}=0 \\
\rho_{0} b \omega \nu_{\alpha} \mu^{\alpha}+\left[-\rho_{0} \frac{\omega^{2}}{k^{2}}+\rho_{0} a_{*}^{2}-i \omega(\lambda+2 \eta)\right] k_{\alpha} v^{\alpha}=0
\end{gathered}
$$

and a system of equations for quantities $\mu_{\perp}^{\alpha}=$ $\left(\delta_{\beta}^{\alpha}-\nu^{\alpha} \nu_{\beta}\right) \mu^{\beta}, v_{\perp}^{\alpha}=\left(\delta_{\beta}^{\alpha}-\nu^{\alpha} \nu_{\beta}\right) v^{\beta}$ :

$$
\begin{aligned}
\left(\beta_{\perp}-i \omega \theta_{\perp}\right) \mu_{\perp}^{\alpha} \mp \frac{i}{2} k M_{0} \theta_{\perp} v_{\perp}^{\alpha} & =0, \\
\pm \frac{k}{2} \beta_{\perp} M_{0} \mu_{\perp}^{\alpha}+\left(\omega \rho_{0}+i \eta k^{2}\right) v_{\perp}^{\alpha} & =0 .
\end{aligned}
$$

The system of equations (17) describes longitudinal waves with the dispersion equation

$$
\frac{\omega^{2}}{k^{2}}=a_{*}^{2}-\frac{\rho_{0} b^{2}}{4 \pi+\beta_{\|}-i \omega \theta_{\|}}-i \frac{\omega}{\rho_{0}}(\lambda+2 \eta) .
$$

The velocity and disturbances of the magnetization vector and the magnetic field strength vector for these waves are directed along the wave vector

$v^{\alpha}=V \frac{k^{\alpha}}{k} \frac{\rho_{1}}{\rho_{0}}, \quad \mu^{\alpha}=-\frac{1}{4 \pi} h^{\alpha}=-\nu^{\alpha} \frac{b}{4 \pi+\beta_{\|}-i \omega \theta_{\|}} \rho_{1}$.

The system of equations (18) describes transverse waves with the dispersion equation

$$
\omega \rho_{0}+i k^{2}\left(\eta+\frac{1}{4} \frac{M_{0}^{2} \beta_{\perp} \theta_{\perp}}{\beta_{\perp}-i \omega \theta_{\perp}}\right)=0,
$$

for which $\rho_{1}=h^{\alpha}=0, k_{\alpha} \mu^{\alpha}=k_{\alpha} v^{\alpha}=0$. The velocity vector in this wave is oriented arbitrary in the plane orthogonal to the wave vector.

Now let us consider the case when $\sin \psi_{0} \neq 0\left(k^{\alpha} \neq\right.$ $\left.\pm k \nu^{\alpha}\right)$. Contracting the equations (16) with the vector components $s_{\alpha}=[\boldsymbol{k}, \boldsymbol{\nu}]_{\alpha}$ by the index $\alpha$ we obtain a system of two equations for quantities $s_{\alpha} v^{\alpha}, s_{\alpha} \mu^{\alpha}$, describing the transverse wave

$$
\begin{gathered}
\left(\beta_{\perp}-i \omega \theta_{\perp}\right) s_{\alpha} \mu^{\alpha}-\frac{i}{2} M_{0} \theta_{\perp} k \cos \psi_{0} s_{\alpha} v^{\alpha}=0, \\
\frac{1}{2} \beta_{\perp} M_{0} k \cos \psi_{0} s_{\alpha} \mu^{\alpha}+\left(\omega \rho_{0}+i \eta k^{2}\right) s_{\alpha} v^{\alpha}=0 .
\end{gathered}
$$

From equations (21) we obtain the dispersion equation for transverse wave

$$
\omega \rho_{0}+i k^{2}\left(\eta+\frac{1}{4} \frac{M_{0}^{2} \beta_{\perp} \theta_{\perp}}{\beta_{\perp}-i \omega \theta_{\perp}} \cos ^{2} \psi_{0}\right)=0 .
$$

For this transverse wave the disturbances of mass density and the magnetic field strength vector are equal to zero, and the velocity vector and the magnetization vector disturbances are orthogonal to the wave vector and magnetization vector

$$
\rho_{1}=h^{\alpha}=0, \quad k_{\alpha} \mu^{\alpha}=\nu_{\alpha} \mu^{\alpha}=0, \quad k_{\alpha} v^{\alpha}=\nu_{\alpha} v^{\alpha}=0 .
$$

For low frequencies, when quantity $\omega \theta_{\perp}$ can be neglected, the wave penetration depth $\delta$ is determined by effective viscosity $\mu_{e}$ :

$$
\delta=\sqrt{2 \eta_{e} /\left(\rho_{0} \omega\right)}, \quad \eta_{e}=\eta+\frac{1}{4} M_{0}^{2} \theta_{\perp} \cos ^{2} \psi_{0} .
$$

For high frequencies the penetration depth is determined by the common viscosity $\delta=\sqrt{2 \eta /\left(\rho_{0} \omega\right)}$.

If $\cos ^{2} \psi_{0}=1$, then the dispersion equation (22) grades into equations (20).

Contracting equations (16) with the vector components $\nu_{\alpha}, k_{\alpha}$ by the index $\alpha$ we obtain a system of four equations for quantities $k_{\alpha} \mu^{\alpha}, \nu_{\alpha} \mu^{\alpha}, k_{\alpha} v^{\alpha}, \nu_{\alpha} v^{\alpha}$ :

$$
\begin{gathered}
-\frac{i}{2} M_{0} \theta_{\perp} k \cos \psi_{0} k_{\alpha} v^{\alpha}+\frac{i}{2} M_{0} \theta_{\perp} k^{2} \nu_{\alpha} v^{\alpha}+\left(4 \pi \sin ^{2} \psi_{0}\right. \\
\left.+\beta_{\perp}-i \omega \theta_{\perp}\right) k_{\alpha} \mu^{\alpha}-\left(\beta_{\perp}-i \omega \theta_{\perp}\right) k \cos \psi_{0} \nu_{\alpha} \mu^{\alpha}=0, \\
\frac{4 \pi}{k} \cos \psi_{0} k_{\alpha} \mu^{\alpha}+\left(\beta_{\|}-i \omega \theta_{\|}\right) \nu_{\alpha} \mu^{\alpha}+\frac{\rho_{0} b}{\omega} k_{\alpha} v^{\alpha}=0, \\
\quad \frac{1}{2} H_{0} k \cos \psi_{0} \nu_{\alpha} \mu^{\alpha}-\frac{1}{2}\left(H_{0}+4 \pi M_{0} \sin ^{2} \psi_{0}\right) k_{\alpha} \mu^{\alpha} \\
+\left(\omega \rho_{0}+i \eta k^{2}\right) \nu_{\alpha} v^{\alpha}-\left(\omega \rho_{0}+i \eta k^{2}\right) \frac{\cos \psi_{0}}{k} k_{\alpha} v^{\alpha}=0, \\
\rho_{0} b \nu_{\alpha} \mu^{\alpha}+\left[-\rho_{0} \frac{\omega}{k^{2}}+\rho_{0} \frac{a_{*}^{2}}{\omega}-i(\lambda+2 \eta)\right] k_{\alpha} v^{\alpha}=0 .
\end{gathered}
$$

Equations (23) describe waves with dispersion equation

$$
\left(V^{2}-\Delta_{\|}\right)\left(V^{2}-\Delta_{\perp}\right)+\left(\frac{f}{\Delta}\right)^{2}=0
$$

for which $s_{\alpha} \mu^{\alpha}=s_{\alpha} v^{\alpha}=0$. In equation (24) $V$ is the wave phase velocity, coefficients $\Delta_{\|}, \Delta_{\perp}, \Delta$ and $f$ are defined as follows

$$
\begin{aligned}
\Delta_{\|} & =a_{*}^{2}-i \frac{\omega}{\rho_{0}}(\lambda+2 \eta) \\
& -\frac{1}{\Delta} \rho_{0} b^{2}\left(4 \pi \sin ^{2} \psi_{0}+\beta_{\perp}-i \omega \theta_{\perp}\right), \\
\Delta_{\perp} & =-i \frac{\omega}{\rho_{0}}\left(\eta+\frac{1}{4} M_{0}^{2} \theta_{\perp}\right) \\
& +\frac{1}{4 \rho_{0} \Delta} M_{0}^{2} \omega^{2} \theta_{\perp}^{2}\left(4 \pi \cos ^{2} \psi_{0}+\beta_{\|}-i \omega \theta_{\|}\right), \\
\Delta & =\left(\beta_{\|}-i \omega \theta_{\|}\right)\left(\beta_{\perp}-i \omega \theta_{\perp}\right) \\
& +4 \pi\left[\left(\beta_{\|}-i \omega \theta_{\|}\right) \sin ^{2} \psi_{0}+\left(\beta_{\perp}-i \omega \theta_{\perp}\right) \cos ^{2} \psi_{0}\right], \\
f & =\pi b M_{0} \omega \theta_{\perp} \sin \left(2 \psi_{0}\right) .
\end{aligned}
$$

It follows from equations (24) and (25) that phase velocity $V$ of considered waves is a function of angle $\psi_{0}$ between the wave vector and the magnetization vector and is independent of wave number $k$.

Coefficients $\Delta_{\|}, \Delta_{\perp}, \Delta f$ are independent of phase velocity $V$, hence equation (24) is a biquadratic equation for determining the phase velocity.

In the general case, the dispersion equation (24) describes interacting longitudinal and transverse waves. If the wave vector is collinear or orthogonal to the magnetization vector $M_{0}$, or if the transverse relaxation time $\theta_{\perp}=0$ is equal to zero, or if $\theta_{\|}=b=0$, then coefficient $f$ in dispersion equation (24) is equal to zero, and the dispersion equation (24) in this case has two solutions for $V$ describing non-interacting longitudinal and transverse waves with dispersion equations $V^{2}=\Delta_{\|}$and $V^{2}=\Delta_{\perp}$.

The case when the wave vector is directed along the magnetization vector $\left(\sin \psi_{0}=0\right)$ has been considered above. If the wave vector is orthogonal to the magnetization vector $\left(\cos \psi_{0}=0\right)$, then the relationship between 
$\mu^{\alpha}$ and $h^{\alpha}$ in the longitudinal wave is degenerated

$$
h^{\alpha}=0, \quad \mu^{\alpha}=-\nu^{\alpha} \frac{b}{\beta_{\|}-i \omega \theta_{\|}} \rho_{1},
$$

and the dispersion equation for longitudinal wave $V^{2}=$ $\Delta_{\|}$can be represented as

$$
\frac{\omega^{2}}{k^{2}}=a_{*}^{2}-\frac{\rho_{0} b^{2}}{\beta_{\|}-i \omega \theta_{\|}}-i \frac{\omega}{\rho_{0}}(\lambda+2 \eta) .
$$

Equations

$$
h^{\alpha}=0, \quad s_{\alpha} \mu^{\alpha}=k_{\alpha} \mu^{\alpha}=0, \quad s_{\alpha} v^{\alpha}=\nu_{\alpha} v^{\alpha}=0,
$$

are satisfied for this longitudinal wave, i.e. the velocity vector is directed along the wave vector, and the magnetization vector disturbances are directed along vector $M_{0}$.

The dispersion equation $V^{2}=\Delta_{\perp}$ which can be represented as

$$
\omega \rho_{0}+i k^{2}\left(\eta+\frac{1}{4} \frac{M_{0}^{2}\left(4 \pi+\beta_{\perp}\right) \theta_{\perp}}{4 \pi+\beta_{\perp}-i \omega \theta_{\perp}}\right)=0
$$

correspond to a transverse wave for case $\cos \psi_{0}=0$ when the wave is propagated orthogonal to the constant magnetization vector. Equations

$$
\rho_{1}=0, \quad s_{\alpha} \mu^{\alpha}=\nu_{\alpha} \mu^{\alpha}=0, \quad s_{\alpha} v^{\alpha}=k_{\alpha} v^{\alpha}=0,
$$

are satisfied for this transverse wave, i.e. the velocity in this wave is directed along the magnetization vector $\boldsymbol{M}_{0}$, and the magnetization vector disturbances are directed along the wave vector.

If $\cos \psi_{0}=0$, then equations (21) describe two waves. The first transverse wave with the dispersion equation

$$
\omega \rho_{0}+i \eta k^{2}=0
$$

for which $\rho_{1}=0, h^{\alpha}=\mu^{\alpha}=0$, and the velocity vector is orthogonal to the wave vector and magnetization vector $\nu_{\alpha} v^{\alpha}=k_{\alpha} v^{\alpha}=0$. Such a wave is studied in detail in Navier-Stokes viscous-fluid flow theory (see, for example, [11]).

The second wave with the frequency determined by equation

$$
\beta_{\perp}-i \omega \theta_{\perp}=0
$$

for which $\rho_{1}=0, h^{\alpha}=v^{\alpha}=0$, the vector with components $\mu^{\alpha}$ is orthogonal to the wave vector and magnetization vector $\nu_{\alpha} \mu^{\alpha}=k_{\alpha} \mu^{\alpha}=0$, and the wave vector is only limited by condition $\nu_{\alpha} k^{\alpha}=0$.

Thus, at $\cos \psi_{0}=0$ four non-interacting waves are possible - a longitudinal wave with the dispersion equation (26), transverse wave with the dispersion equation (27), a second transverse wave with the dispersion equation (28) and the wave determined by equation (29). One can see from equations $(19),(20),(22),(26)-(28)$ that the longitudinal waves in an ML propagated along the magnetization vector $\boldsymbol{M}_{0}$, or orthogonal to it, do not depend on the transverse magnetization relaxation time $\theta_{\perp}$, and the transverse waves do not depend on the longitudinal relaxation time $\theta_{\|}$.

The velocity of sound for the waves in question is determined by the vector of group velocity with components $\partial \omega / \partial k^{\alpha}$. In contrast to the Neuringer-Rosensweig model (without viscosity), the velocity of sound in the relaxation model depends on the wave frequency. From the dispersion equations (19) and (26) it can be inferred that at low frequencies (as $\omega \rightarrow 0$ ), if the viscosity is neglected, the velocity of sound $a$ at the points $\psi=\pi / 2$ and $\psi=0$ is determined by the relationships:

$$
\left.a^{2}\right|_{\psi=\pi / 2}=a_{*}^{2}-\frac{\rho_{0} b^{2}}{\beta_{\|}},\left.\quad a^{2}\right|_{\psi=0}=a_{*}^{2}-\frac{\rho_{0} b^{2}}{4 \pi+\beta_{\|}} .
$$

At high frequencies (as $\omega \rightarrow \infty$ ) the velocity of sound $a$ at the points $\psi=\pi / 2$ and $\psi=0$ is determined as follows

$$
\left.a^{2}\right|_{\psi=0}=\left.a^{2}\right|_{\psi=\pi / 2}=a_{*}^{2}
$$

\section{Matching with the experimen- tal data}

For convenience in evaluating the experimental data let us represent the internal energy $\rho U_{m}$ as

$$
\rho U_{m}=\rho U_{m}^{0}(\rho, s)+f(\rho, s, M),
$$

where $\rho U_{m}^{0}(\rho, s)$ is the internal energy of a nonmagnetized liquid, and function $f(\rho, s, M)$ is zero for a non-magnetized liquid $f(\rho, s, 0)=0$.

Then for $a_{*}^{2}$ in formula (??) we can write

$$
a_{*}^{2}=a_{0}^{2}+a_{M}^{2},
$$

where the velocity of sound in a non-magnetized liquid $a_{0}$ and quantity $a_{M}$ are determined by the formulas

$$
a_{0}^{2}=\left(\rho \frac{\partial^{2} \rho U_{m}^{0}}{\partial \rho^{2}}\right)_{0}, \quad a_{M}^{2}=\left(\rho \frac{\partial^{2} f}{\partial \rho^{2}}\right)_{0} .
$$

The experiments show $[8,9]$ that for ferrofluids the following statements are confirmed:

1. The velocity of sound when the wave is propagated along the magnetization vector is greater than the velocity of sound when the wave is propagated orthogonal to the magnetization vector.

One can see from the equations (30) and (31) that this condition is met at low and high frequencies if $\beta_{\|}>0$ or $\beta_{\|}<-4 \pi$.

2. The velocity of sound in the magnetized liquid increases as the wave frequency increases.

From definitions (30), (31) we obtain

$$
\begin{gathered}
\left.a^{2}\right|_{\psi=0, \omega \rightarrow \infty}-\left.a^{2}\right|_{\psi=0, \omega \rightarrow 0}=\frac{\rho_{0} b^{2}}{\beta_{\|}}, \\
\left.a^{2}\right|_{\psi=\pi / 2, \omega \rightarrow \infty}-\left.a^{2}\right|_{\psi=\pi / 2, \omega \rightarrow 0}=\frac{\rho_{0} b^{2}}{\left(4 \pi+\beta_{\|}\right)} .
\end{gathered}
$$

From equations (34) it follows that at $\beta_{\|}>0$ the inequality $\left.a^{2}\right|_{\omega \rightarrow \infty}>\left.a^{2}\right|_{\omega \rightarrow 0}$ is fulfilled both when the wave is propagated along the magnetization vector $M_{0}$, and orthogonal to vector $M_{0}$. Thus, at $\beta_{\|}>0$ the velocity of sound at high frequencies is greater than the velocity of sound at low frequencies. From definitions (??), (32) it follows that the condition $\beta_{\|}>0$ sets the following limitation to function $f$ :

$$
\beta_{\|}=\left(\frac{\partial^{2} f}{\partial M^{2}}\right)_{0}>0
$$


3. The velocity of sound increases as the liquid is magnetized. Because of items 1 and 2 it is sufficient to consider this issue for low frequencies when the wave is propagated orthogonal to the magnetization vector. In this case the wave velocity $\left.a\right|_{\psi=\pi / 2}$ according to equations (30), (33) is determined by the formula

$$
\left.a^{2}\right|_{\psi=\pi / 2}=a_{0}^{2}+a_{M}^{2}-\frac{\rho_{0} b^{2}}{\beta_{\|}} .
$$

In accordance with the experiment, the value of $\left.a^{2}\right|_{\psi=\pi / 2}$ should be greater than the value of $a_{0}^{2}$ in a non-magnetized liquid. Hence, the inequality

$$
\left.a^{2}\right|_{\psi=\pi / 2}-a_{0}^{2}=a_{M}^{2}-\frac{\rho_{0} b^{2}}{\beta_{\|}}>0
$$

should be satisfied. It sets another limitation on function f:

$$
\left(\frac{\partial^{2} f}{\partial \rho^{2}}\right)_{0}\left(\frac{\partial^{2} f}{\partial M^{2}}\right)_{0}>\left(\frac{\partial^{2} f}{\partial \rho \partial M}\right)_{0}^{2} .
$$

Inequalities (35), (36) should be also satisfied in the Neuringer-Rosensweig model of a ferrofluid.

\section{REFERENCES}

[1] J. L. Neuringer, R. E. Rosensweig. Ferrohydrodynamics, Phys. Fluids. Vol.7, No.12, 1927-1937, 1964.

[2] M. I. Shliomis. Magnetic fluids, Sov. Phys. Usp. Vol.17, 153-169, 1974.

[3] V. A. Zhelnorovich. On models of magnetizale and polarizable media with microstructure, Doklady Akad.
Nauk. SSSR Vol.249, No. 2, 333-337, 1979.

[4] V. A. Zhelnorovich. Models of Continuous Media with Internal Electromagnetic and Mechanical Moments, Moscow state univ., Moscow, 1980 [in Russian].

[5] J. L. Snoek. Time effects in magnetisation, Physica, No. 5, 663-668, 1938.

[6] L. I. Sedov. On the ponderomotive forces of interaction of an electromagnetic field and an accelerating material continuum, taking into account finite deformations, Prikl. Mat. Mekh. (Journal of Applied Mathematics and Mechanics) Vol.29, No. 1, 4-17, 1965.

[7] V. A. Naletova. Lectures on Ferrohydrodynamics, Moscow state univ., Moscow, 2005 [in Russian].

[8] J. P. McTague. Magnetoviscosity of magnetic colloids, Chem. Phys. Vol.51, No. 1, 133-136, 1969.

[9] V. M. Polunin. Acoustic Effects in Magnetic Fluids, Fizmatlit, Moscow, 2008 [in Russian].

[10] V. A. Zhelnorovich. Magnetoacoustic Waves in magnetizable liquids, Technical Physics, Vol.57, No.1, 149-153, 2012.

[11] L. D. Landau, E. M. Lifshitz. Fluid Mechanics, Pergamon Press, New York, 1987. 